\title{
Acceso a la información pública gubernamental: ¿incentivo para la mejora administrativa? (Evaluación de una política pública transversal, frente a la gobernanza)
}

\author{
Access to governmental public information: \\ Incentive for administrative improvement? \\ (Evaluation of a cross-cutting public policy in the face of governance)
}

DOI: $10.46814 / \operatorname{lajdv} 3 n 5-030$

Recebimento dos originais: 01/05/2021

Aceitação para publicação: 31/06/2021

\author{
Martín Cutberto Vera Martínez ${ }^{1}$ \\ Profesor investigador. Universidad Autónoma de Baja California, México \\ E-mail: martin.vera@uabc.edu.mx
}

\section{RESUMEN}

El presente artículo presenta un análisis pormenorizado de la evolución administrativa y legal de las políticas públicas del acceso a la información gubernamental en México y la evaluación de su vinculación con la modernización y mejora de la administración pública y el combate a la corrupción. Se sostiene que, si bien la rendición de cuentas ha generado gran cantidad de datos y se considera como un factor indispensable para la democracia, sus resultados están orientados al margen del cambio administrativo y la disminución del desorden y el abuso en la función pública, como lo muestra la persistencia de las tasas de corrupción administrativa y política.

Palabras clave: Transparencia, acceso a la información pública, corrupción, reforma administrativa, políticas públicas.

\begin{abstract}
This paper presents a detailed analysis of the administrative and legal evolution of public policies on access to government information in Mexico and evaluation of its relationship with the modernization and improvement of public administration and fighting corruption. It is argued that while accountability has generated large amounts of data and is considered as an essential factor for democracy, their results are oriented outside the administrative change and the decline of disorder and abuse in the public service, as show the persistence rates of administrative and political corruption.
\end{abstract}

Keywords: Transparency, access to public information, corruption, administrative reform, public policies.

\footnotetext{
${ }^{1}$ Doctor en Gobierno y Administración Pública por el Instituto Universitario de Investigación José Ortega y Gasset, Universidad Complutense de Madrid, España. FEyRI, Calzada Universidad 14418, Parque Industrial Internacional Tijuana, Tijuana, B.C., México, C.P. 22427
} 


\section{INTRODUCCIÓN}

Desde los tiempos de Aristóteles y su relato sobre La Política, se ha destacado la importancia de la evolución de la democracia en un marco de libertad, en donde los ciudadanos puedan tener acceso a juzgar los asuntos públicos, con el fin de generar un equilibrio de las fuerzas políticas. Sin embargo, nuestra hipótesis es que el acceso a la información pública enfocado a la transparencia y a la participación ciudadana no implica necesariamente la existencia de sistemas y procesos eficaces en sí mismos, para transformar el comportamiento de la administración pública, si no son acompañados o integrados por otros factores necesarios para el logro de tales fines.

\section{DEFINICIÓN Y FACTORES DEL ACCESO A LA INFORMACIÓN PÚBLICA.}

En la filosofía política, un desarrollo importante en los procesos democráticos en las últimas dos décadas ha sido el renacimiento de la democracia deliberativa (Elster, 1998). En tal sentido, el derecho a la información constituye un instrumento fundamental para el desarrollo de la democracia. En ella, cada ciudadano puede observarse como propietario de una cuota del patrimonio público (Fuenmayor, 2004).

Este concepto permite inferir que el rol de dicho acceso tiene como fin que la información fluya «hacia abajo» para que los gobernados puedan conocer, evaluar y supervisar las acciones de los gobernantes, y así ejercer una suerte de control social «hacia arriba» (Nassens, 2010).

\section{El concepto de transparencia y su relación con la información pública}

Aunado al derecho de acceso a la información, encontramos el concepto de transparencia, el cual, como vertiente del primero, se ve motivada a estructurarse, por tener el atractivo de la promesa de abatimiento de la corrupción, de optimizar la eficiencia de las instancias gubernamentales y de, sustantivamente, mejorar la calidad de vida de las personas, al tener éstas la posibilidad de utilizar las leyes y las instancias administrativas, como herramienta para nutrir de mejores contenidos la toma cotidiana de decisiones (Madero, 2003). En este orden de ideas, cuando la transparencia se encuentra orientada hacia el ejercicio de la administración pública, hace referencia a valores sociales como la honestidad, la claridad, la participación y el respeto de reglas tanto para el gobernante como para el gobernado (Bellver, 2007).

Florini (1999), aporta su visión al ejercicio de la transparencia, refiriéndose a actos tales como "la divulgación, por parte de las instituciones, de información que es relevante para evaluarlas". Así entendida, la transparencia permite dicha difusión de información, como un insumo relevante para la evaluación de los organismos públicos y en tal sentido es un tema que ha generado gran interés para las ciencias sociales contemporáneas, entendido por su posible contribución al logro de un régimen de administración eficaz y creíble. 
La transparencia puede dividirse en dos grandes grupos de ejecución. Por un lado, existen aquellas políticas, llamadas también de primera generación, que apuntan a abrir los archivos oficiales o de acceso a la información pública y cuya expresión más conocida la constituyen las leyes de acceso a la información (Dassen y Vieyra, Ob. Cit.). Proponen, básicamente, una dinámica verticalista que sostiene que si se tiene acceso a la información pública, los ciudadanos contarán con las herramientas fundamentales para controlar mejor lo que hacen los gobiernos, fortalecer la participación ciudadana y la democracia moderna. (Rose-Ackerman, 1999).

Por otra parte, el otro grupo de políticas de transparencia denominadas de segunda generación, o transparencia focalizada, tienen como objetivo "la divulgación de una información específica, identificando y diagnosticando un objetivo político preciso para la divulgación de la información" (Dassen et al., 2012). Sin embargo, cabe destacar que dicha información, aunque pueda ser disponible y accesible, al existir la posibilidad de que no sea comprendida por quien accede a ella, puede tener como resultado desinformar o bien diferir de la que se esperaría obtener a través de una política de acceso a nivel general (Graham, 2002).

\section{EVOLUCIÓN DEL SISTEMA DE ACCESO A LA INFORMACIÓN PÚBLICA.}

El derecho a la información se incluyó en la Constitución por primera vez en México, en 1997, en el marco de la denominada "reforma política", en donde se modificó el Artículo $6^{\circ}$ constitucional para añadir el texto que reza “el derecho a la información será garantizado por el Estado”, un precepto complementario del Artículo $8^{\circ}$ que ya reconocía el derecho de petición ${ }^{2}$. Sin embargo, tal garantía individual careció de soporte en la legislación secundaria hasta la promulgación de la Ley Federal de Transparencia y Acceso a la Información Pública Gubernamental (LFTAIPG), publicada en el Diario Oficial de la Federación (DOF) el 11 de junio de $2002^{3}$. Además, se creó el Instituto Federal de Acceso a la Información Pública, como un órgano de la Administración Pública Federal, con autonomía operativa, presupuestaria y de decisión, cuyo objetivo radicó, en esa etapa, en promover y difundir el ejercicio del derecho de acceso a la información, resolver la negativa a las solicitudes de información

\footnotetext{
${ }^{2}$ Si bien es cierto que durante muchos años existió el derecho de petición, reconocido por el Artículo $8^{\circ}$ de la Constitución, éste sólo obligaba a la autoridad a emitir acuerdo por escrito con respecto a una solicitud, presentada por un particular. Tal acuerdo podía negar una solicitud de información, argumentando que era un "secreto administrativo", o que la autoridad no la tenía, o que carecía de facultades para divulgarla. La ausencia de respuesta, por regla general, se consideraba una negativa ficta.

${ }^{3}$ Para la elaboración de la ley fueron tomadas en cuenta diversas iniciativas como la del diputado Miguel Barbosa; la del presidente Vicente Fox y los grupos parlamentarios del PRD, PRI, PT, PVEM y Convergencia Democrática, elaborada por el Grupo Oaxaca. Ver Escobedo, Juan Francisco. Movilización de opinión pública en México: el caso del Grupo Oaxaca y de la Ley Federal de Acceso a la Información Pública, Derecho comparado de la información, México: Instituto de Investigaciones Jurídicas, 2003.
} 
y proteger los datos personales en posesión de las dependencias y entidades de la Administración Pública Federal.

El 5 de julio de 2010 se publicó por decreto la denominación del "Instituto Federal de Acceso a la Información Pública" por la de "Instituto Federal de Acceso a la Información Pública y Protección de Datos". A la ley federal, le siguieron leyes equivalentes en las entidades federativas. Para finales de 2007 todos los estados de la república contaban con una ley en la materia, aunque sus criterios y normas variaban notablemente. De igual forma el Plan Nacional de Desarrollo 2001-2006 tenía como objetivo construir lo que denominó un "gobierno abierto y transparente".

Gráfico 1.

Año de publicación de las Leyes del País

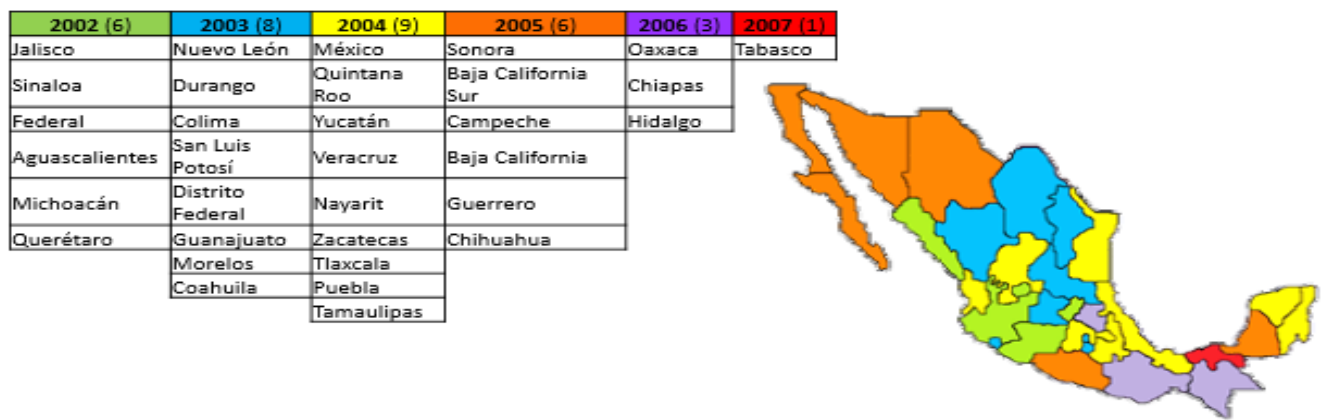

Fuente: Elaboración del autor con datos derivados del IFAI.

De acuerdo con Ernesto Villanueva (2003), el derecho de acceso a la información pública se ha convertido en parte de la agenda de la reforma del Estado en varios países de América Latina, no siendo México la excepción. Por otro lado, la práctica de este derecho hizo notoria una serie de obstáculos administrativos que lo volvían nugatorio ante los ciudadanos. ${ }^{4}$ Por ello, llegaron a llamarse "leyes de máxima reserva" de la información (Guerra, 2001).

Una nueva reforma, en 2007, establece el estándar mínimo de su ejercicio y sienta los principios de la política de transparencia gubernamental ${ }^{5}$, con lo que la siguiente etapa, en la reforma del 7 de febrero de $2014^{6}$, se provee de autonomía constitucional al IFAI, dando inicio a una nueva etapa en la evolución del acceso a la información en el país.

\footnotetext{
${ }^{4} \mathrm{~A}$ modo de ejemplo, se solicitaba para el ejercicio del derecho la obligación de estampar una firma autógrafa en la solicitud y en el recurso de revisión, así como la acreditación de personalidad del solicitante, la imposibilidad de presentar solicitudes vía electrónica, grandes causales de reserva y falta de especificación en caso de incumplimiento.

${ }^{5}$ Para un análisis detallado del contenido de la reforma constitucional véase, entre otros, Bustillos y Carbonell (2007). Hacia una democracia de contenidos: la reforma constitucional en materia de transparencia. México: Instituto de Investigaciones Jurídicas de la Universidad Autónoma de México. Carbonell, Miguel, 2008, El régimen constitucional de la transparencia. México: UNAM; Salazar, Pedro (2008). El derecho de acceso a la información en la Constitución Mexicana. Razones, significados y consecuencias. México: UNAM-IFAI.

${ }^{6}$ Asimismo, el reglamento para la IFAI como organismo autónomo fue publicado el 20 de febrero de 2014, disponible en http://www.dof.gob.mx/nota_detalle.php?codigo=5333316\&fecha=20/02/2014
} 
En los avances que se han registrado entre 1998 a 2013 para crear una cultura de la transparencia y de rendición de cuentas se inscriben tres grandes vertientes: el robustecimiento del control interno de cada uno de los tres poderes federales, la neutralización del sistema (los tres poderes empiezan a vigilarse mutuamente) y el avance en la capacidad de la ciudadanía para acceder a la información generada por el gobierno, en concordancia con procesos similares que se replican internacionalmente.

\section{ANÁLISIS DEL OBJETIVO, MISIÓN, VISIÓN Y EVOLUCIÓN INSTITUCIONAL DEL ACCESO A LA INFORMACIÓN.}

No obstante los avance enumerados, si bien la rendición de cuentas ha generado gran cantidad de datos y se considera como un factor indispensable para la democracia, todavía no se han hallado los medios para cristalizar su efectividad, así como tampoco se observa a la percepción de la transparencia, como un valor institucional consolidado por parte de la ciudadanía ${ }^{7}$.

Desde 1998, la transparencia y el acceso a la información pública representan no sólo un avance en la sociedad de la información mexicana, sino también supone ser un medio de lucha contra la corrupción y de avance democrático (García López, 2007). En este nuevo desarrollo institucional se enmarcó la creación del Instituto Federal de Acceso a la Información y Protección de Datos (IFAI) Hoy Instituto Nacional de Acceso a la Información Pública y Protección de Datos (INAI), que pronto se validó como rector innegable de la política pública de apertura de la información generada por el gobierno ${ }^{8}$.

La tendencia en la consolidación del número de solicitudes que recibe anualmente este organismo confirma esta afirmación, como se observa en la siguiente gráfica, cuya finalidad es básicamente observar la tendencia creciente de la demanda:

\footnotetext{
${ }^{7}$ Los estudios de Latinobarómetro dan cuenta detallada de la baja confianza ciudadana en el conjunto de las organizaciones públicas. Ver http://www.latinobarómetro.org

${ }^{8}$ Para mayor información sobre INAI ver: https://home.inai.org.mx
} 
Gráfico 2

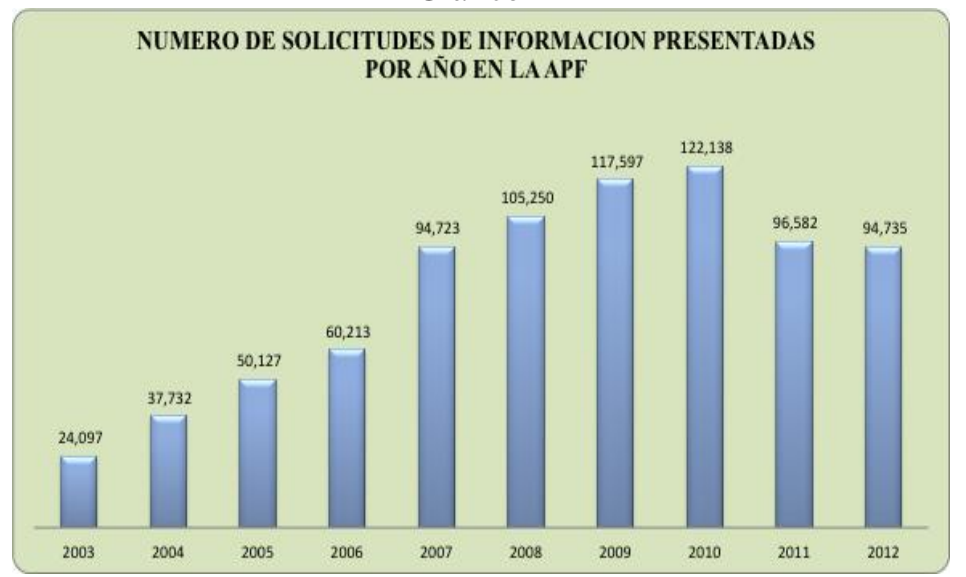

Fuente: Elaboración propia, con información de https//:home.inai.org.mx

Es de reiterarse que, a pesar de que el sistema de acceso a la información pública gubernamental permite identificar numerosas acciones de corrupción en la gestión lo mismo de recursos humanos, materiales, financieros, obra pública, contratos, uso de facultades, etcétera, su efecto final es sólo de divulgación publicitaria, ya que el diseño del sistema lo concibe como un fin en sí mismo y no como un medio para transformar las estructuras y fuentes de origen de las desviaciones en el seno de los organismos públicos.

En la exposición de motivos de la ley vigente, se señalaron objetivos explícitos de impacto final en el núcleo de la corrupción, a saber: • Establecer un modelo administrativo abierto y democrático en contraposición al modelo cerrado y "criptográfico" anterior, •La eficiencia administrativa a través de la rendición de cuentas y la supervisión ciudadana; y $\bullet$ La reducción de la corrupción ${ }^{9}$. Es decir, el acceso a la información se concibe como un medio orientado a un fin superior. Incluso, en dirección diferente al avance alcanzado en el sistema institucional, se preveía en la motivación de su diseño como política pública del estado, buscar que de oficio se pusiera al alcance de los ciudadanos un conjunto de información, tanto detallada como agregada, que permitiera conocer las actividades del Estado de forma permanente, sin que fuera necesaria solicitud alguna, con simpleza y certeza funcional, no necesariamente administrada o sobre administrada, como es hoy día.

Para corroborar su fin último, se puede señalar que para evaluar su impacto, hasta ahora, el principal indicador es uno solo, el "Indicador de tiempo de respuesta a solicitudes de información y calidad de las mismas (ITRC)", muy lejano de cualquier consideración de impacto en la modificación del comportamiento o adecuación de las instituciones públicas.

\footnotetext{
${ }^{9}$ Éstos objetivos son detallados con profusión en la iniciativa enviada como proyecto al Poder Legislativo por el Presidente de la república, en su parte de exposición de motivos
} 
Inclusive, la lectura de la Misión del instituto reafirma estas consideraciones, al señalar que su objetivo principal es, textualmente "Garantizar el derecho de los ciudadanos a la información pública gubernamental y a la privacidad de sus datos personales, así como para promover en la sociedad y en el gobierno la cultura del acceso a la información, la rendición de cuentas y el derecho a la privacidad", conjuntamente con su Visión, al plantearse alcanzar "mecanismos que garanticen que las resoluciones de los comisionados sean efectivamente inatacables, haciendo hincapié en que parte importante de esta acción es asegurar que la información que se entrega sea veraz y completa".

Todo ello, se aleja incluso, de preceptos de la ley señalados en su artículo 2, especialmente en dos importantes apartados, que tienen que ver con el objetivo que hemos estado mencionando de que la transparencia debe ser causal de mejora de la administración pública y de la eficacia contra la corrupción. Los mismos son:

"VII. Promover, fomentar y difundir la cultura de la transparencia en el ejercicio de la función
pública, el acceso a la información, la participación ciudadana, asi como la rendición de
cuentas, a través del establecimiento de politicas públicas y mecanismos que garanticen la
publicidad de información oportuna, verificable, comprensible, actualizada y completa, que
se difunda en los formatos más adecuados y accesibles para todo el público y atendiendo en
todo momento las condiciones sociales, económicas y culturales de cada región”"

y “VIII. Propiciar la participación ciudadana en la toma de decisiones públicas a fin de contribuir a la consolidación de la democracia". Indudablemente, se trata de conceptos clave "cultura de transparencia en la función pública" y la "consolidación de la democracia".

En ese tenor, tienen más obligaciones los sistemas estatales de transparencia, ya que deben cumplir con más indicadores de gestión, tales como publicidad de actuación, publicidad de informes de labores, datos de presupuesto, quejas, nivel académico de los comisionados, entre otros, pero ninguno vinculado al impacto final del sistema, como mecanismo de combate a la corrupción y cambio administrativo.

Con relación a dichos sistemas subnacionales, es de señalarse que incluso, demuestran no solo debilidad en la orientación a la transparencia y la lucha contra la corrupción, sino cierta tendencia a dificultar el acceso a la misma y, posiblemente, a encubrir sus actos, toda vez que de acuerdo con el Índice Nacional de Órganos Garantes del Derecho de Acceso a la Información (INOGDAI), creado por Article 19 y México Infórmate, para 2019, el 81\% de los institutos estatales encargados del acceso al derecho a la información no lo garantizan y en cambio, ponen trabas para la entrega de datos, no transparentan los argumentos para negar las solicitudes hechas por ciudadanos o no cuentan con un sistema de procedimientos para el manejo de información ${ }^{10}$.

\footnotetext{
${ }^{10}$ Existe información amplia de estas mediciones en http://www.inogdai.org/?inicio/objetivo/0
} 
A modo de reflexión, podemos destacar que si bien el acceso a la información pública se encuentra orientado a la transparencia y a la participación ciudadana, sólo será un medio eficaz contra la corrupción, cuando sea acompañada de un diseño institucional con elementos favorables para un efectivo impacto de las acciones de publicidad y la rendición de cuentas del gobierno, complementadas con el desarrollo y la capacitación de sociedades civiles; el fortalecimiento de la administración pública; mecanismos de intermediación y vinculación con reformas en las fuentes identificadas de corrupción, ineficacia e ineficiencia administrativa.

\section{ANÁLISIS DEL FENÓMENO DE LA CORRUPCIÓN, QUINQUENIO DE 1998 A 2013.}

Aristóteles es el primero en utilizar la palabra corrupción, misma que invoca para referirse a la degeneración que alcanzan las formas de gobierno monárquico, aristocrático y democrático, derivándose de su génesis las formas corruptas son respectivamente tiranía, oligarquía y demagogia. Della Porta y Vanucci (2002) la definen como "aquellas acciones u omisiones que tienen que ver con el uso abusivo de los recursos públicos para obtener beneficios privados”. Es decir, estaremos en presencia de un acto de corrupción cuando se produzca una violación de alguna norma -ya sea por acción u omisión- establecida por funcionarios o actores sociales encargados de vigilar, gestionar o administrar los recursos públicos.

Además del soborno y la extorsión, otras formas de corrupción incluyen acuerdos recíprocos en desmedro del público, interpretaciones tendenciosas en la aplicación de las normas legales, favoritismo, clientelismo, uso de información privilegiada y tráfico de influencias.

Para ayudar a los gobiernos, sectores privados y, sobre todo, otros interesados en el tema en el seno de la sociedad civil, se ha creado un organismo no-gubernamental llamado Transparency International $^{11}$ (TI) para combatir la corrupción a través de una red de Capítulos en más de 90 países. Dicha entidad publica el Índice de Percepción de Corrupción (IPC). Dicho indicador es una "encuesta de encuestas" basadas, a su vez, en diversas encuestas de opinión pública (formuladas por el Banco Mundial, la Universidad de Columbia o el Foro Económico Mundial, entre otros) sobre la magnitud de la corrupción en 120 países, que refleja las percepciones de empresarios, analistas de riesgo, especialistas financieros y otros, de la situación de la corrupción en cada país. Además del elaborado por TI, existen diferentes índices entre los que podemos mencionar: Barómetro Mundial de la Corrupción (basado fundamentalmente en percepciones del sector privado); Índice Fuentes de Soborno

\footnotetext{
${ }^{11}$ A nivel internacional, el movimiento TI tiene como objetivo principal infundir al sistema global de valores la transparencia y la rendición de cuentas como normas públicamente reconocidas. Trabaja con el sector privado y organizaciones internacionales tales como la OCDE, para fortalecer el marco político y legal de los negocios internacionales. Para mayor información consultar http://www.transparency.org/
} 
(percepción que se tiene de las empresas más importantes que operan internacionalmente); Índice de Opacidad. Realizado por PriceWater House Coopers (basado en encuestas individuales identifica prácticas poco transparentes principalmente en la esfera administrativa; el sistema legal y las políticas económicas entre otras); Encuesta Nacional de Corrupción y Buen Gobierno (registra la frecuencia de los actos de corrupción para la obtención de servicios públicos en las 32 entidades federativas del país y en los 38 servicios públicos); Encuesta Nacional de Cultura Política y Prácticas Ciudadanas (ENCUP) de la Secretaría de Gobernación (diagnostica rasgos de la cultura política y de las prácticas ciudadanas predominantes).

De acuerdo con los informes de TI, a través de su Índice de Percepción de la Corrupción, México se encuentra entre los países más corruptos y en los últimos 23 años ha descendido vertiginosamente, del lugar 55 en 1998 al 124 en $2020^{12}$.

El país aparece sistemáticamente con niveles que indican una alta proliferación de prácticas corruptas y la población señala que las acciones emprendidas por el Presidente en turno en contra de la corrupción son "pocas" o "regulares" (Arellano, 2012). Casos multi nombrados como el del ex gobernador del Estado de México, Arturo Montiel quien, tras finalizar su cargo, se descubrió que acrecentó su fortuna desde los 2 millones de pesos mexicanos a 111 millones, o empresas mexicanas que pagan cada año 8400 millones de dólares en sobornos a legisladores y otros funcionarios públicos para influir en el diseño y la aplicación de las leyes o la financiación de campañas electorales (incluyendo el escándalo de Vicente Fox), la Biblioteca José Vasconcelos, el caso TURISSSTE (subcontratación de eventos por una ganancia de 1 mil 862 millones de pesos), FOVISSSSTE (otorgamiento de créditos hipotecarios irregulares por 570 millones de pesos), licitación y compra de medicamentos por 307 millones de pesos por parte de entidades federativas, adquisición de la vacuna de influenza AH1N1 por un perjuicio al público de 117.9 millones de pesos, la Estela de Luz, ó la adjudicación de fideicomisos por 1.051 millones de pesos, entre tantos otros, se han prestado también al planteamiento de sospechas y dudas sobre el uso y origen de los dineros públicos y privados. Asimismo, Entre los países latinoamericanos, su situación como país no es más favorable, como se muestra gráficamente enseguida.

${ }^{12}$ El desglose y la comparativa de resultados se puede consultar con mayor amplitud en https://www.tm.org.mx/ipc2020/ 
Gráfico 3

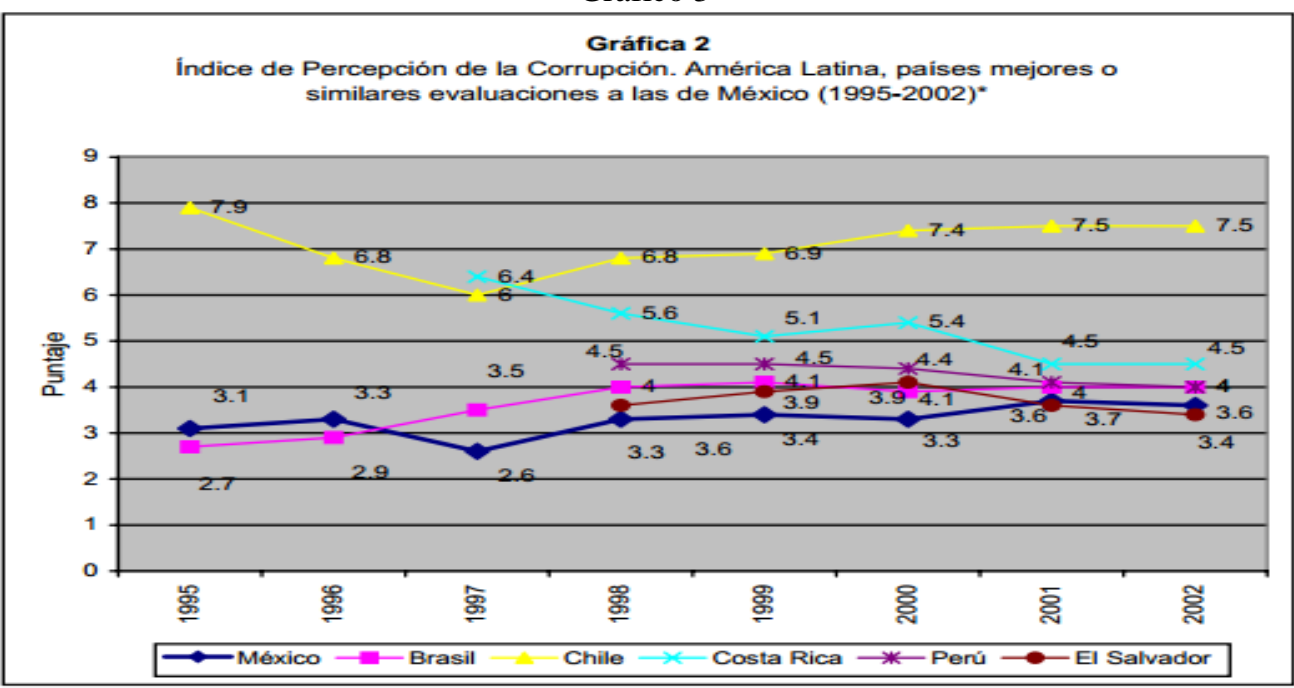

Fuente: Transparency International, www.transparency.org * $10=$ altamente limpio; $0=$ altamente corrupto

Lo cierto es que, como puede observarse en los gráficos anteriores, a la par de la consolidación del sistema de acceso a la información pública, la corrupción crece exponencialmente, creando una contradicción institucional entre ambas políticas públicas.

Los estudios disponibles indican que la tolerancia hacia estas prácticas alienta su extensión a otros campos y acarrea consecuencias aún más dañinas; contribuye a crear atmósferas de inseguridad en los negocios (pues la certeza comprada con un soborno es inestable); y genera grupos de interés con una gran capacidad para oponerse a las reformas que el Estado debe realizar para combatir la corrupción. Según encuestas realizadas por organismos internacionales y nacionales existe una alta permisividad entre la población hacia prácticas corruptas y deshonestas.

Otra de las dolencias que han permitido a la corrupción ganar terreno en México es la impunidad. Hay estados donde el delito prescribe antes de que se termine de auditar la cuenta pública: en algunas entidades el margen es de menos de dos años, en otros en el mejor caso, cinco. La evidencia indica que las políticas anticorrupción requieren la adopción de una estrategia más integradora que la simple formulación de emitir declaraciones, denuncias políticas o legislación.

Sendos estudios han demostrado también que cuando en México se dio información a los votantes sobre los niveles de corrupción, la población respondió con apatía política y se presentó en menores cantidades a votar, o bien que los usuarios de la información simplemente no confían en que sus reclamos acerca del accionar del sector público sean suficientes para cambiar su comportamiento y resolver problemas históricos (UNESCO, 2012).

La rendición de cuentas constituye una exigencia ubicua en el mundo de la política democrática (Schedler, 1999). Abarca de manera genérica tres maneras diferentes para prevenir y corregir abusos de poder: obliga al poder a abrirse a la inspección pública; lo fuerza a explicar y justificar sus actos y 
lo supedita a la amenaza de sanciones. Involucra por tanto el derecho a recibir información y la obligación correspondiente de divulgar todos los datos necesarios, recibir una explicación y el deber correspondiente de justificar el ejercicio del poder. Ahora bien, si el Estado Mexicano ha llevado adelante políticas de transparencia tendentes a brindar a los ciudadanos la posibilidad de controlar actos corruptos cabe preguntarse entonces ¿Existe una relación entre transparencia y corrupción? ¿Han impactado las políticas de gobierno abierto la disminución de los casos de corrupción? ¿El acceso a la información pública se ha convertido en un sistema administrativo más?

\section{ANÁLISIS DE LA INCIDENCIA DEL SISTEMA DE INFORMACIÓN PÚBLICA Y REFORMA ADMINISTRATIVA.}

Arellano (2000) señala que darle contenido práctico a la transparencia, como política organizativa, implica comprender las tensiones múltiples que se generan y no considerarlo meramente como un simple instrumento que pueda ser de inmediato incorporado al hardware organizativo. Así, la transparencia debiera ser interpretada como un engranaje de relaciones entre los grupos y los individuos, racionalizado y hecho razonablemente en el conjunto de valores y de normas de la acción organizativa. Sin embargo, en México los alcances y límites del control externo e interno a los cuales se encuentra expuesto el Poder Ejecutivo son frágiles (Guerrero, 2003); “ni los índices del IPC (por el lado de la demanda de corrupción) ni los del BPI (fuentes u oferta de sobornos) referidos anteriormente, muestran evidencias perceptibles de mejoras desde que se han estado publicando periódicamente" (Scholoss, 2013), así como tampoco los indicadores de percepción de la corrupción indican avances en el combate a la corrupción (salvo una mayor concientización sobre el tema), no obstante el número creciente de convenciones, convenios internacionales, aprobación de nuevas legislaciones en diversos países, etc.

El acceso a la información se reconoce por el volumen de recursos o solicitudes presentadas, pero dicho reconocimiento no resulta extensivo en el impacto en el mejoramiento de la administración pública, aunque originalmente estuviere planteado para ello. Es decir, constituye un círculo que gira sobre sí mismo. El acceso a la información se justifica por el mismo acceso a la información, no por su capacidad de incidir en la mejora del funcionamiento del gobierno y de los funcionarios públicos.

Existen diferentes factores para explicar lo antedicho. En primer lugar, puede obedecer a un complejo abordaje jurídico de la política a llevar a cabo, lo cual se verá reflejado en un exceso de aspectos formales que, por lo general, son lentos, burocráticos y de mucha menor flexibilidad que la que se requiere para hacer frente a la destreza, versatilidad e imaginación de los agentes que practican la corrupción (Schloss, 2013). En segundo lugar, cuestiones de carácter sociológico también imponen ciertas limitaciones; por ejemplo, la falta de apropiación de los mecanismos por parte de la sociedad, 
pueden considerarse como un fenómeno negativo al desarrollo de la legitimidad del derecho de acceso y reclamo de respuestas. Finalmente, una percepción ética y moral negativa de la función pública actúa como elemento revelador del buen funcionamiento del Estado (Guerrero, Ibid). Tal como señala Prats (2007), la falta de valores y su vinculación con la corrupción causan el deterioro de la credibilidad y la imagen de lo público, recayendo sobre ella la sospecha de dar cobijo a conductas poco lícitas o de subyacentes intereses espurios alejados del valor público. Ahora bien, si los ciudadanos no sólo desean servicios eficientes y de calidad, sino que también exigen moral, ética y valores cívicos ¿la rendición de cuentas es un fin en sí mismo para tales demandas o tan sólo una herramienta político-jurídica?

En términos generales, se admite como verdadera la relación causal establecida entre transparencia y corrupción; esto es, a mayor transparencia del ejercicio de funciones y a mayor acceso a la información pública, es menor el grado de corrupción vigente en una sociedad determinada. En tal sentido, el diseño y la ejecución de políticas y programas públicos aceptan (en el sentido de no cuestionarlo) y fundan su existencia, en la validez de dicha relación, pero dejan por fuera el mecanismo de evaluación de los efectos que provocan los comportamientos de las organizaciones, unidades o personal de la administración pública respecto de la corrupción; es decir, no se comprueba la relación de causalidad en sí misma, sino que sólo se la refiere para justificarla como herramienta aplicable para el fin último que radica en la reducción de la corrupción. Sin embargo, si los resultados arrojados por los diferentes índices que miden el fenómeno de la corrupción en México no reflejan tal correspondencia, es posible inferir que existan otros factores que deban ser tomados en cuenta para poder cumplir con los resultados esperados. En palabras de Aguilar-Bustelo (2010) "el ejercicio estándar de evaluación no cuestiona la estructura causalidad (el teorema, el modelo y la tecnología) de la política y del programa, la supone correcta". En consecuencia puede afirmarse que los valores de eficacia, eficiencia y receptividad, promovidos por las leyes y políticas de transparencia no configuran, por sí solos o necesariamente, un impacto positivo en la reducción de la corrupción y en consecuencia, un gobierno ético y responsable.

Consideramos que para que lo último se cristalice, sería necesaria una evaluación del diseño de las propias políticas y estructuras que promueven la transparencia, así como también de la idoneidad de la organización que las implementa y los resultados de la acción pública, a fin de poder examinar con detenimiento "la relación causal que contiene el diseño de la política pública, el tipo de organización pública que es responsable de su implementación y el modo de gobernanza o proceso de gobernar, ya que la contingencia de la organización, puede o no asegurar la implementación y gestión eficaz de las políticas, programas y servicios” (Aguilar-Bustelo, Ibid). 
Analizar la gestión de políticas orientadas a la reducción de la corrupción únicamente desde un enfoque gubernamentalista ${ }^{13}$, y no de verdaderas políticas públicas, considerando que un acto corrupto obedece a un crimen de cálculo y oportunidad sin ninguna relación con otros factores, implicaría incurrir en un reduccionismo que no termina de responder a nuestro interrogante inicial, es decir, la necesidad de identificar otras herramientas para incidir positivamente en un resultado concreto que reduzca los índices de corrupción.

"La transparencia de la información como criterio, entonces, implica establecer marcos específicos de comparación y medición de las actividades organizativas, con el fin de establecer parámetros más o menos medibles que permitan a los actores organizacionales calcular los costos y beneficios de su interacción, así como compartir el sentido de la acción y la posibilidad de construir nuevos valores que permitan dar sentido a la transparencia de una forma internalizada y no sólo como respuesta a un castigo o a una amenaza (Arellano, Ibid).

México ha concluido con relativo éxito su proceso de transición a la democracia, pero lo que se exige ahora son resultados por medio del afianzamiento de instituciones de rendición de cuentas sujetas a evaluación, tanto en el ámbito federal como en los gobiernos estatales y municipales. De otra forma, la democracia mexicana puede convertirse en un concepto vacío al no producir gobiernos eficaces y honestos (Ugalde, 2000). Existen varios vehículos para evaluar las áreas concretas de vulnerabilidad como pueden ser las encuestas sistemáticas para "triangular" y evaluar cada una de las áreas de gobierno vulnerables a la corrupción (Wamey, 1999) ${ }^{14}$ o ciertas medidas dentro del modelo de gobernanza del Estado y de la sociedad civil, como sería "entender y diagnosticar cómo funciona el sistema corrupto específicamente en el contexto particular del país o región; superar la resistencia política y burocrática, y generar apoyo de los que pueden generar cambios; así como crear un plan de acción secuencial para superar los sistemas corruptos, romper la cultura del cinismo y generar ímpetu político y de transformación de gobierno, sociedad civil y sectores empresariales”(Schloss, Ibid). Un modelo de gobernanza ${ }^{15}$ y su consecuente evaluación se encontraría entonces orientado a incluir otros factores y variables tanto intra estatales como societales, sustentando la conducción política y

\footnotetext{
${ }^{13}$ El enfoque gubernamentalista sostiene la idea de que el gobierno es suficiente en la solución de los problemas sociales y en la producción de los objetivos sociales preferidos, por lo que tanto el éxito como el fracaso relativo es atribuible exclusivamente al gobierno y a sus organismos.

${ }^{15}$ Cabe distinguirlo del término gobernabilidad, entendida como la estabilidad y orientación de la conducción política del Estado, preservando la estabilidad y la cohesión, pero desde la perspectiva del propio Estado. Según este enfoque, la gobernabilidad considera que el problema directivo es suficientemente resuelto si se dota o se provee de nuevo al gobierno con las capacidades requeridas (instituciones, fiscales, administrativas, coactivas...) para estar en aptitud de dirigir a la sociedad. Para un desarrollo analítico sobre la gobernanza nos permitimos remitir a Aguilar, L.F. Gobernanza y Gestión Pública. México: Fondo de Cultura Económica, 2006; Natera, A., La noción de gobernanza como gestión pública participativa y reticular. Documentos de Trabajo Política y Gestión 2. Madrid: Universidad Carlos III de Madrid, 2004.
} 
democrática del Estado, con estabilidad y cohesión, pero desde la perspectiva de la base social o de la ciudadanía. En palabras de Aguilar-Bostelo (Ibidem) "la insuficiencia gubernamental para definir y sobre todo realizar los futuros preferidos de sociedad hace que los gobiernos requieran y valoren las capacidades de la sociedad económica y civil, particularmente en los asuntos de crecimiento económico y bienestar o seguridad social universal, lo cual introduce otro tipo de relación entre el gobierno y la sociedad y obliga al gobierno a practicar otro modo de gobernar a la sociedad". También se estima necesario repensar los alcances y límites del control interno o autocontrol del Poder Ejecutivo para avanzar hacia un auténtico equilibrio entre poderes en México, ya que los controles externos a los que está expuesto el Ejecutivo son todavía frágiles e insuficientes ${ }^{16}$.

Para crear una estructura acompañada de incentivos para que los funcionarios públicos y los propios ciudadanos se involucren en las acciones correctivas, es necesario comprender las causas y los costos que los actos de corrupción traen consigo. Seguidamente, debieran trazarse metas a corto, mediano y largo plazo que prevean: a) la selección de los agentes de cambio; b) la determinación de los incentivos, recompensas y penalizaciones; c) medición de los resultados; d) y la reestructuración de la relación entre los actores principales, los agentes de cambio y los beneficiarios: reducir el monopolio, clarificar y limitar discreción y mejorar la rendición de cuentas (Schloss, Ibidem).

Con relación al factor social como variable incidente en la configuración de políticas de transparencia, los itinerarios del proceso general de modernización, planteados por las teorías funcionalistas sostienen que la modernización social deviene luego de una modernización política económica. ${ }^{17}$ En ese entorno, las sociedades modernas, debido al alto grado de especialización funcional, son sociedades sin centro y sin estructura dominante capaz de regular enteramente las relaciones intrasistémicas y sociales, lo cual hace cada vez más difícil la posibilidad de ordenar coherentemente las funciones de cada sistema parcial en atención a una lógica general de la sociedad y a un vértice constituido para darle dirección a tal lógica. Es por ello que estimamos que la coordinación de políticas anticorrupción, sin la debida integración sistémica no puede brindar resultados favorables.

\footnotetext{
${ }^{16}$ La identificación de los problemas no siempre conlleva a métodos sofisticados: en las etapas iniciales de la acción correctiva una estrategia más intuitiva es muchas veces suficiente para por lo menos detectar las áreas "más obvias" de disfunción. Ciudadanos, empresarios y funcionarios públicos que trabajan en instituciones sistemáticamente corruptas pueden analizar dónde y cómo se posiciona la corrupción. Esto puede ser sorprendente, pero muchas veces este es el caso, con tal que el enfoque sea orientado hacia el sistema corrupto y no a los individuos.

${ }^{17}$ No son pocas las evidencias que indican el desajuste en el movimiento permanente de las tres dimensiones y una exigencia de coordinación sobre la base de una marcada diferenciación de expectativas y lógicas de operación
} 


\section{CONCLUSIONES}

Este artículo si bien ha demostrado la importancia de la transparencia como alternativa en la lucha contra la corrupción, ha tenido como fin contribuir a la reflexión y explicación de las razones por las cuales este vínculo no necesariamente genera una disminución en tales actos ilícitos. En materia de transparencia, el escenario actual en México presenta mecanismos favorables para el acceso a la información desde la aprobación de la Ley Federal de Transparencia y Acceso a la Información Pública y la consecuente obligación de las dependencias del gobierno federal a abrir a la sociedad todos sus archivos, documentos e información. Sin embargo, la transparencia de información pública y el conocimiento de determinados datos no ha resultado efectivo para la disminución de la clasificación del país como favorable a perpetrar actos corruptos por parte de sus funcionarios.

Consideramos que la visión del potencial de la transparencia como instrumento para la mejora de las instituciones políticas y sociales no debe hacer referencia a políticas públicas aisladas sino, más bien, a diferentes partes de un abordaje sistémico del problema de la corrupción. Por ello sugerimos que, si se tiene como fin el sustentar un modelo de gobernanza y un método de su evaluación con éxito, además de la legitimidad institucional, al cumplimiento de la legalidad y a la consecución de resultados, se deben añadir -y por lo tanto definir- por una parte, la capacidad directiva de los gobiernos para conducir la compleja red de agencias, personas y valores intraburocráticos.

La relación entre la transparencia y la corrupción es objeto de dos importantes condiciones: la publicidad y la rendición de cuentas. Sin embargo, la mera puesta de la disposición de información ante los ciudadanos sin mecanismos sancionadores no resulta propicia para prevenir la corrupción.

A su vez, consideramos que cualquier propuesta para obtener los efectos buscados no debe dejar de lado los factores culturales, puesto que, en definitiva, tanto dentro como fuera de las instituciones públicas, son los seres humanos quienes conducen los grupos sociales. Se trata de evidenciar los antagonismos relacionados con la cultura que el país debe superar a fin de consolidar, desde adentro hacia afuera y desde abajo hacia arriba, un dilema cultural que en muchos casos se encuentra en vías de transformación pero que en otros, todavía no ha despertado el cambio. Esto se puede sintetizar en cuatro grandes disyuntivas, a saber: a) cultura del secreto vs. cultura de la rendición de cuentas: lo cual implica involucramiento y reclamos por parte de los destinatarios interesados a fin de lograr el efecto buscado; b) cultura de la votación vs. cultura de la participación: es decir, superar la visión según la cual la participación en la democracia por parte del ciudadano equivale solamente a la votación en las elecciones y la percepción de la ciudadanía que estima la existencia de una baja probabilidad de que su participación en la vida cívica produzca un cambio de comportamiento en el sector administrativo; c) cultura de la ignorancia de la cosa pública vs. cultura del conocimiento, lo que indica que si las necesidades desean verse traducidas en demandas, el actor debe estar en conocimiento 
de una serie de procedimientos, modalidades de acción y rituales propios de la acción políticoinstitucional. y lograr apropiarlos; d) cultura individual vs. cultura colectiva, según la Encuesta Mundial de Valores organizada por la Universidad de Michigan ${ }^{18}$, la confianza interpersonal está estrechamente vinculada con la democracia, con el respeto a la ley y con el asociacionismo, es decir que la buena administración no es nada concedido por la gracia de los gobernantes si no un derecho que va conquistándose por la ciudadanía activa y organizada.

Según nuestra tesis, toda política de desarrollo que promueva reformas institucionales centradas únicamente en el aumento de la transparencia resulta insuficiente para el fin que se predispone a combatir. Si deseamos que la transparencia funcione al servicio de la lucha contra la corrupción la mera detección de los abusos no basta. Un sistema también necesita autoridades con la capacidad y mando para actuar según la información y denuncias recibidas en la reforma administrativa correspondiente.

Peter Eigen (1995), presidente de TI, ha conceptualizado el dilema: ¿Cuán creíble puede ser esperar que una maquinaria gubernamental corrupta -sin hablar de la que lo es de forma sistemáticase reforme a sí misma?" Su respuesta a esta pregunta es previsible: no es verosímil que un sistema corrupto se "purifique" por generación espontánea. La participación y las políticas públicas son parte de un cambio sistémico e integrado de la organización estatal, que permitiría producir una reingeniería del sistema de acceso a la información, para que se vinculen y se midan los cambios en los procesos administrativos y el impacto en la corrupción en general, como el componente central del sistema de rendición de cuentas, anulando la reincidencia de las conductas y los sistemas que producen la corrupción que por ahora, solo se publicitan.

\footnotetext{
${ }^{18}$ Para más información acerca de la Encuesta Mundial de Valores, vea www.worldvaluessurvey.org
} 


\section{REFERENCIAS}

Aguilar, Luis y Bustelo, María. 2010. "Gobernanza y evaluación: una relación potencialmente fructífera". Gestión y Análisis de Políticas Públicas. Instituto Nacional de Administración Pública (4), pp. 3-10. Recuperado de http://www.redalyc.org/articulo.oa?id=281521696002

Arellano, David. 2000. Reformando al gobierno: una visión organizacional del cambio gubernamental. México: Porrúa.

Arellano, Efrén. Impacto de la corrupción en la desconfianza política, La cultura de la corrupción en México. Recuperado de www3.diputados.gob.mx/...PB4004\%20Impacto\%20de\%201a\%20corrupcion. Consulta 14 de agosto de 2014.

Bellver, Ana. 2007. "Reformas en materia de transparencia: segunda generación de cambio institucional”. Revista del CLAD Reforma y Democracia, Volumen 38.

Dassen, Nicolás y Vieyra, Juan. 2012. “Gobierno abierto y transparencia focalizada”. Banco Interamericano Desarrollo. 4-10. Recuperado de: http://www.iadb.org/es/temas/transparencia/apoyoa-los-paises/publicacion-gobierno-abierto-y-transparencia-focalizada, 7365.html

Della Porta, Donatella y Vanucci, Alberto. 2002. "Los recursos de la corrupción: algunas reflexiones sobre el caso italiano". Dialnet, (98) pp. 85-118. Recuperado de http://dialnet.unirioja.es/servlet/articulo?codigo=247726

Doyle, Kate. 2003. “Comentarios sobre la Ley Federal de Transparencia y Acceso a la Información Pública Gubernamental”. Derecho Comparado de la Información, (2), 163-172.

Eigen, Peter. 1995. Ética, responsabilidad y transparencia: la teoría puesta en práctica. Recuperado de www.inap.mx/portal/.../etica\%20y\%20administracion\%20publica.pdf

Elster, Jon. 1998. "Deliberation and Constitution Making". Deliberative Democracy. (pp. 97-118). Cambridge: Cambridge University Press.

Florini, Ann. 1999. "Does the Invisible Hand Need a Transparency Glove?" The Politics of Transparency. Annual World Bank Conference on Development Economics. 28-30. Recuperado de http://web.worldbank.org/WBSITE/EXTERNAL/WBI/EXTWBIGOVANTCOR/0,,contentMDK:208 00998 isCURL:Y menuPK:1977057 pagePK:64168445 piPK:64168309 theSitePK:1740530,00.ht $\mathrm{ml}$

Fuenmayor, Alejandro. 2004. El derecho de acceso de los ciudadanos a la información pública. Oficina de la UNESCO para Centroamérica, pp. 11-25. Recuperado de http://portal.unesco.org/es/file_download.php/561ff4bc2719856c5184270296fc48f5EL+DERECHO +DE+ACCESO+DE+LOS+CIUDADANOS+A+LA+INFORMACION+PUBLICA.pdf

García, Genaro. 2007. "El acceso a la información de la administración pública en los Estados Unidos Mexicanos: la regulación del principio de libre información”. Scielo. 21, (43), p.112-120. Recuperado de http://www.scielo.org.mx/scielo.php?script=sci_arttext\&pid=S0187358X2007000200005\&lng=es\&n $\mathrm{rm}=\mathrm{iso}>$. ISSN 0187-358X. 
Graham, Mary. 2002. Democracy by Disclosure, the Rise of Technopopulism. Washington D.C.: Brookings Institution Press.

Guerra, Oscar M. 2001. Los órganos garantes de transparencia. Integración y características: su funcionamiento como órganos autónomos. México: Instituto de Investigaciones Jurídicas de la Universidad Nacional Autónoma de México.

Guerrero, Eduardo. 2003. "Cultura de transparencia y rendición de cuentas en la gestión pública. Instituto Federal Electoral", $\quad$ 12-20. http://www.ife.org.mx/documentos/DECEYEC/ensayo_7.pdf

Madero, Francisco. 2003. "Cultura de transparencia y rendición de cuentas en la gestión pública. Instituto Federal Electoral", $\quad$ pp. 21-29. Recuperado de http://www.ife.org.mx/documentos/DECEYEC/ensayo_7.pdf

Nassens, Hilda. 2010. "Ética Pública y Transparencia”. Centro de Investigación en Ciencias Sociales y Humanidades. Universidad Autónoma del Estado de México, pp. 2113-2129. Recuperado de http://halshs.archives-ouvertes.fr/docs/00/53/15/32/PDF/AT15_Naessens.pdf

Prats, Joan. 2007. "La lucha contra la corrupción como parte integrante del Derecho, el deber y las políticas de buena administración”. Cuadernos de Derecho Público (31), 1-47. Recuperado de http://bci.inap.es/material-la-lucha-contra-la-corrupci\%C3\%B3n-como-parte-integrante-del-derechoel-deber-y-las-pol\%C3\%ADticas-de

Rose-Ackerman, Susan. 1999. Corruption and Government: Causes, Consequences, and Reform. Cambridge: Cambridge University Press.

Schedler, Andreas. 2008. ¿Qué es la rendición de cuentas? Cuadernos de Transparencia IFAI, (3). Recuperado de www.redalyc.org/pdf/599/59918310.pdf

Scholoss, Miguel H. 2013. "Experiencias Internacionales para fortalecer la gobernabilidad. Revista Gobierno y Gestión Pública", pp. 8-10. Recuperado de http://gobiernoygestionpublica.edu.pe/revista_digital/pdf/1_2.pdf

Ugalde, Luis C. 2000. "La Rendición de Cuentas en los Gobiernos Estatales y Municipales". Auditoría Superior de la Federación, (4) pp. P. 42-45. Recuperado de www.asf.gob.mx/uploads/63_serie_de_rendicion_de_cuentas/rc4.pdf

Villanueva, Ernesto. 2003. "Derecho de acceso a la información en el Poder Judicial. Una aproximación al caso mexicano desde la perspectiva comparada". Revista Mexicana de Justicia, (2), p.207-212.

Villoria, Manuel. 1999. "El papel de la burocracia en la transición y consolidación de la democracia española: primera aproximación”. Revista Española de Ciencia Política, volumen 1, (1) 121-123.

Wamey, Julius. 1999. Can Corruption be Measured? Bank's World. Recuperado de www.worldbank.org/wbi/governance/diag_surveys.htm 